\title{
Endobronchial mass lesion due to sarcoidosis: complete resolution with corticosteroids
}

\author{
BRUCE F CORSELLO, GERHARD H LOHAUS, AKIRA FUNAHASHI \\ From the Department of Medicine, Veterans Administration Medical Center, Milwaukee, Wisconsin, and the \\ Department of Medicine, Medical College of Wisconsin, Milwaukee, Wisconsin, USA
}

Sarcoidosis is a granulomatous disorder of unknown aetiology affecting many systems, including the lungs and mediastinal lymph nodes. The usual manifestations of sarcoidosis on the chest radiograph are well known. Although the bronchial mucosa is often affected in sarcoidosis, endobronchial mass lesions are extremely rare. ${ }^{1}$

Endobronchial lesions are most often due to bronchogenic carcinoma and less often to relatively benign causes such as bronchial adenoma, lipoma, broncholith, and tuberculosis. ${ }^{2-6}$ We report the first case of a distinct endobronchial mass lesion due to sarcoidosis that resolved with corticosteroid treatment.

\section{Case report}

A 37-year-old black man was admitted to the medical service on 25 May 1981 with a three-day history of feverishness and non-productive cough. Right-lower-lobe infiltrates were seen on the chest radiograph. The patient remained afebrile and examination of the sputum specimen was unremarkable. A diagnosis of non-bacterial pneumonia was made and he was discharged on oral erythromycin.

Inquiry revealed that the patient had been admitted to another hospital in September 1977 for evaluation of bilateral hilar lymphadenopathy, which was thought to be due to sarcoidosis. Transbronchoscopic lung biopsy specimens at that time showed non-caseating granulomas but no endobroncial lesions were seen. Corticosteroids were not prescribed.

One month later dry cough and right-lower-lobe infiltrates persisted and the patient underwent flexible fibreoptic bronchoscopy on 29 June 1981. The bronchial mucosa was generally inflamed, much more severely on the right side. An endobronchial mass lesion was noted at the orifice of the apical segmental bronchus of the right lower lobe, with nearly complete occlusion. A small amount of purulent material was initially noted in the narrowed bronchus. The lesion was smooth and broad based, with slightly increased vascularity which at first glance resembled a bronchial adenoma (fig 1). Biopsy specimens of this lesion, however, showed multiple non-caseating granulomas, as

Address for reprint requests: Dr Bruce F Corsello, Department of Medicine/111E, Veterans Administration Medical Center, 5000 West National Avenue, Wood (Milwaukee), Wisconsin 53193, USA. did additional mucosal biopsy specimens from the rightupper-lobe bronchus. Cultures of bronchoscopic specimens for mycobacteria and fungi were negative. The diagnosis of endobronchial sarcoidosis was made and the patient was started on oral prednisone, $60 \mathrm{mg}$ daily, on 29 July 1981. On 26 August 1981 he was symptom free and his chest radiograph showed complete resolution of the previous right-lower-lobe infiltrates. Prednisone was gradually decreased. Repeat flexible fibreoptic bronchoscopy on 23 September 1981 showed considerable improvement of mucosal inflammation and a complete resolution of the previously observed endobronchial lesion (fig 2). Multiple mucosal biopsy specimens from the orifice to the apical segmental bronchus of the right lower lobe, as well as from the right-upper-lobe bronchus, showed only moderate inflammation and no granulomas were seen. The patient was maintained on decreasing doses of oral prednisone until 17 March 1982, when this medication was discontinued. When last seen on 2 June 1982 he had no respiratory complaints and had a completely normal chest radiograph.

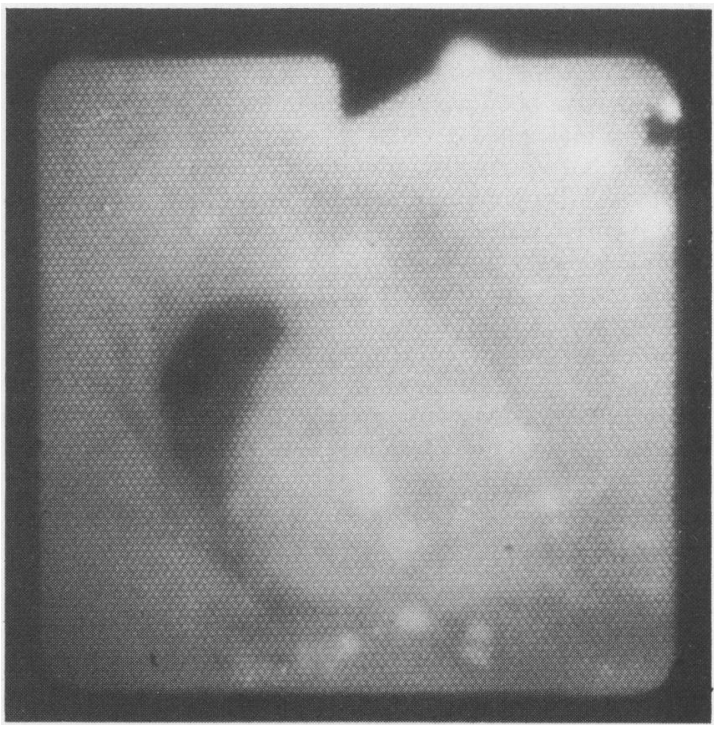

Fig 1 Bronchoscopic view of endobronchial mass lesion, showing a nearly complete occlusion of the orifice. 


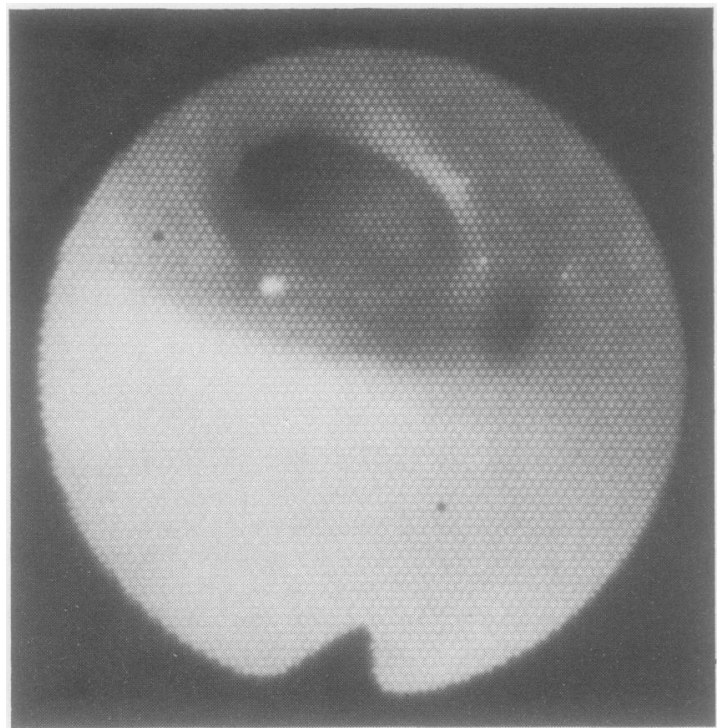

Fig 2 Bronchoscopic view showing a complete resolution of the previous endobronchial mass lesion.

\section{Discussion}

The diagnosis of sarcoidosis was firmly established in this case. Bilateral hilar lymphadenopathy was noted on the chest radiograph in September 1977 and the transbronchoscopic lung biopsy specimens showed non-caseating granulomas. Although the bilateral hilar lymphadenopathy had resolved by May 1981, the mucosal biopsy specimens from the endobronchial mass lesion, as well as from the right-upper-lobe bronchus, showed multiple non-caseating granulomas, with negative mycobacterial and fungal cultures.

The frequent lesions of the bronchial mucosa in sarcoidosis have been well documented and biopsy of apparently normal mucosa often shows a microscopic sarcoid process. ${ }^{1}$ Gross mucosal abnormalities are, however, less common. ${ }^{17}$ Projections into the bronchial lumen have been described as "wart-like excrescences"' or "bleb-like elevations 2 to $3 \mathrm{~mm}$ in diameter." The distinct isolated mass lesion seen in the present case has not been previously described. Multiple or isolated bronchostenotic areas with distal atelectasis are also uncommon. These are due to external compression by enlarged lymph nodes or intrinsic narrowing secondary to mucosal thickening rather than distinct, isolated mass lesions. Olsson and associates have reported eight cases of bronchostenosis due to sarcoidosis. ${ }^{8}$ In their series five patients showed isolated broncho- $\stackrel{\oplus}{+}$ stenosis, four of whom had distal atelectasis. Three cases? were treated with corticosteroids but had no clinical or 흐 histological improvement. The major difference between $\frac{\bar{D}}{D}$ the present case and Olsson's series is that our patient had $\mathbb{Q}$ a distinct endobronchial mass lesion which caused bronchial obstruction and distal pneumonitis. Furthermore, his 5 endobronchial lesion did resolve completely after two $\overrightarrow{0}$ months of treatment with oral corticosteroids.

The persistence of new localised infiltrates or atelectasis $\vec{\omega}$ on a chest radiograph should raise the possibility of endo- $\Omega$ bronchial obstruction. In patients with known or suspected $\overrightarrow{\vec{x}}$ sarcoidosis this endobronchial obstruction may be due to $\dot{\omega}$ endobronchial sarcoidosis. Flexible fibreoptic bronchoscopy should be performed with transbronchial biopsies. Oral corticosteroids may be tried if the endobronchial pro- $\vec{V}$ cess is histologically proved to be sarcoidosis, with negative mycobacterial and fungal cultures. An important point is $\supset$ that, unlike most of the earlier reported cases of endobron- $\overrightarrow{ }$ chial sarcoidosis, in the present case there were no physical $\mathbb{T}$ findings or radiographic abnormalities suggesting an active 을 sarcoid process at the time of our initial bronchoscopy. Unnecessary resectional lung surgery might have been $₹$ undertaken had the endobronchial mass lesion been diag- $\vec{\bullet}$ nosed as bronchial adenoma on the basis of gross endo- $\infty$ scopic appearance.

\section{References}

${ }^{1}$ Friedman $\mathrm{OH}$, Blaugrund SM, Siltzbach LE. Biopsy of the bronchial wall as an aid in diagnosis of sarcoidosis. JAMA 1963;108:646-50.

${ }^{2}$ Burcharth F, Axelsson C. Bronchial adenomas. Thorax $\overline{\overline{0}}$ 1972;27:442-9.

${ }^{3}$ Abert T, Blondal T, Nou E, Malmaeus J. The choice of $\vec{P}$ operations for bronchial carcinoids. Ann Thorac Surg 1981;32:19-21.

${ }^{4}$ Politis J, Funahashi A, Gehlsen JA, et al. Intrathoracic lipomas. Report of three cases and a review of the $\frac{O}{\partial}$ literature with emphasis on endobronchial lipoma. $J \times$ Thorac Cardiovasc Surg 1979;77:550-6.

${ }^{5}$ Kelly WA. Broncholithiasis. Postgrad Med 1979;66:8190.

${ }^{6}$ Albert RK, Petty TL. Endobronchial tuberculosis progressing to bronchial stenosis. Chest 1976;70:537-9.

${ }^{7}$ Kalbian VV. Bronchial involvement in pulmonary sarcoidosis. Thorax 1957;12:18-23.

${ }^{8}$ Olsson T, Bjornstad-Petterson H, Stjernberg NL. Bron-을 chostenosis due to sarcoidosis. A cause of atelectasis and airway obstruction simulating pulmonary neop- $\sigma$ lasm and chronic obstructive pulmonary disease. Chest $N$ 1979;75:663-6. 